\title{
Relational Listening: Fostering Effective Communication Practices in Diverse Organizational Environments
}

\author{
Judi Brownell
}

Cornell University

Interest in managing workforce diversity in the hospitality industry has grown steadily over the past several decades. Women, for example, are entering service industries and moving into managerial positions at an unprecedented rate (Del Sesto, 1993); the percentage of older workers has also risen (DeMicco \& Reid, 1988; Sillies, DeMicco, Kavanaugh, \& Mann, 1994). Furthermore, the introduction of legislation in compliance with the Americans with Disabilities Act presents new challenges as innovative programs are put into place to accommodate disabled employees (Woods \& Kavanaugh, 1992; Smith, 1992). As our world becomes a global village, members of the hospitality workforce will require skills and attitudes that foster understanding and collaboration between individuals with different values and perspectives (Christensen, 1993; Gamio \& Sneed, 1992; Griffin, 1992; Mill, 1994; Powers, 1992).

In the past, organizational diversity has referred to groups with distinctive needs and talents. However, categories created for the "new workers" of the 1980s - the disabled, the older worker, women, and cultural minorities_-will become less useful as individuals are recognized not lor their membership in a minority group, but for their unique contributions to the organization (Gerber, 1990; Greenslade, 1991). As the year 2000 approaches, managers will witness a movement toward recognizing unique personal qualities in all organizational members. Consequently, the ability to effectively communicate with individuals who have different values, 
backgrounds, needs, and roles will become much more critical than seeking to determine the special characteristics of any specific minority group.

Hospitality educators and managers have an opportunity and an obligation to foster the key communication competencies needed in the decades ahead, and to ensure that organizational diversity is valued, not just managed. In this paper, relational listening is presented as a means of promoting positive attitudes toward diversity as well as encouraging effective communication practices within diverse organizational environments. Relational listening is facilitated by (1) an understanding of the transactional nature of communication, (2) the skills of empathic listening, and (3) the practice of self-monitoring. Each of these topics is addressed in light of its contribution to cross-cultural communication. It is further proposed that traditional training sessions are less effective in fostering appropriate attitudes and behaviors than more personal, individual approaches such as modeling, coaching, and appraising.

\section{The Communication Challenges of Organizational Diversity}

Each person has a unique framework for viewing the world. To a large extent, employees see what they expect to see given their organizational role, background, and personal values. While an older worker may find a recent memo announcing the introduction of a new computer system threatening and disruptive, a recent hospitality graduate may view the change as an opportunity to show off his or her newly acquired college skills. Upon hearing of an employee party, a young bachelor may look forward to networking with managers from other departments, while his female, Native American co-worker may anticipate a situation that is both awkward and stressful. Such differences are particularly apparent when employees from different cultures 
communicate, and because individuals interpret information in keeping with their backgrounds and previous experiences.

We begin with the premise, that no two people can ever have exactly the same experience and, therefore, their interpretations of a situation are never identical.

Regardless of how well you think you know someone or how similar your experiences may be, you can never completely duplicate another person's viewpoint. When the dimension of diversity is added, we can assume that sharing meanings becomes even more problematic.

The concept of quality service, for instance, may be interpreted differently by different employees. An individual who has previously worked in a four- star resort may have a very different expectation than one who has been a waiter at a fast food restaurant. An employee who has just moved to New York City from the West Indies may have very different understandings about service than a colleague who was trained in the European service tradition. Strong organizational norms developed through a variety of formal and informal management activities may be necessary to keep these employees moving in the same direction toward a shared understanding of service standards.

Given the communication challenges of a diverse workplace, relational listening skills are proposed as one means of facilitating mutual understanding and healthy relationships. You will see that the relational model emphasizes attitudes and skills that foster cross-cultural understanding and the full development of all human resources (Figure 1). Each dimension presented in this paper - the transactional perspective, empathy, and self-monitoringcontributes to more productive workplace encounters. 


\section{A Transactional Perspective}

Transactional models of communication emphasize what goes on between individuals communicating rather than creating and delivering messages. Focus is not only on the speaker's choices regarding content and delivery, but equally on the way in which meanings from the messages are perceived and interpreted by the listener.

A good example of the transactional perspective can be seen as the food and beverage manager prepares to present a new concept to the hotel's executive board. Although she gives the usual care and consideration to defining her purpose and polishing her delivery, her most critical concern is with seeking as much information as possible about her listeners. What are each person's attitudes toward the topic? What do board members already know about the issues involved? How will the recommended changes affect each person?

In interpersonal contexts, arbitrary distinctions between sender and receiver, or speaker and listener, are discarded; participants are seen as simultaneously sending and interpreting verbal and nonverbal cues. In addition, the receiver assigns his or her own meaning, regardless of what the sender intends. If, for instance, you, as a manager, recommend that a spare room be converted to an employee lounge, your intention might well be to improve the workplace. At least one employee, however, may interpret your message to mean that employees are only given space no one else wants, at management's convenience.

Logically, you may assume that communication is facilitated by similarities between participants and hindered or blocked by differences in values, attitudes, and beliefs. In the past, this has been the common view (Figure 2). The transactional perspective, however, suggests instead that similarities between communicators are not essential. Obviously, the more similar individuals' experiences are, the easier it is for them to communicate with one another. If you 
and a colleague have both worked in a French pastry shop, it's likely you have developed a specialized vocabulary and set of competencies. Such common experience, however, is not essential for good communication.

What is required for effective communication is the willingness and the ability to create a third dimension, a unique view somewhat different from that held by either of the participants. When two communicators hold different assumptions and have very different past experiences, they must agree to collaborate in the communication process by negotiating their understandings. As they interact, they move as close as possible to sharing their unique interpretations. In this process, listening emerges as a key activity.

A relational orientation is particularly appropriate for managing in diverse hospitality environments. This is because it facilitates active participation and collaboration while developing common understandings that, although shared, are likely to be somewhat different from the original meanings held by either participant (Figure 3). Communication becomes synonymous with dialogue; the transactional model implies that communicators work together to achieve mutual understanding.

During a performance appraisal, for example, a manager may suddenly realize that one of his Asian employees had no idea that she was not perceived as delivering "friendly" service. In her view, she was courteous and respectful; in light of the performance expectations at this southern California resort, however, the behavior was perceived as formal and aloof. Clearly, the manager could have repeated, "be more friendly," for months and no change would have occurred because his employee had a different understanding of what the concept meant.

Taken within the context of cross-culture encounters, it becomes clear that this framework has profound implications for how managers and employees approach their 
communication tasks. Focus is shifted from generating the necessary information and delivering it with conviction to considering the other person's background, position, and possible response. The service employee and guest, the server and chef, the sales and front office managers - each person participates in a joint effort to better understand the other. The transactional view provides a framework for viewing the employee as a unique communicator, and appreciating individual differences as a natural and anticipated aspect of organizational communication.

The context in which communication occurs is also important. Common contexts are developed over time as individuals have repeated exposure to people in certain roles, settings, and circumstances. Leaders who develop strong organizational cultures have succeeded in creating a common context that all organizational members use to interpret daily events. What does it mean when the general manager repeatedly stops by the housekeeping office and asks, "How are things going?" Is this part of her normal routine, or should it be interpreted as an indication that she suspects a problem?

The transactional model places listening behavior at the very heart of communication as participants engage in a collaborative effort to move process, a third dimension is created that recognizes the interpretations of both participants. This listener- centered model facilitates communication in diverse organizational environments by focusing on the unique attributes of each employee. In addition, two skills also have implications for relational listening: empathy and self-monitoring.

\section{The Nature of Empathy}

Empathy has been defined in a variety of ways (Bruneau, 1989). Generally, it has three distinct dimensions: cognitive, perceptive, and behavioral. All three are important to our 
understanding of relational listening, since each has implications for how employees develop relationships with those whose backgrounds and perceptions are different from their own.

\section{Cognitive aspect}

Empathy occurs when the individual attempts to view a situation as he or she perceives the other person sees it. Instead of thinking, "That's really a stupid thing to do," someone demonstrating empathy would instead try to look at the situation from the other's perspective and think, "She feels alienated and misunderstood. She thinks he was making fun of her cane; her first impulse was to strike out. I understand how she must feel. Although I don't agree with her actions, when I think of it how hard it must be to get around this lobby, her behavior makes sense."

\section{Perceptive aspect}

Empathy refers to an individual's sensitivity to another's nonverbal communication and his or her general awareness of the communication situation. For example, you might think to yourself, "Tom says he's not upset, but I know he just heard about the change in the schedule this morning and, with four children, he will certainly be affected. He's tried to call home several times, and has been drinking one cup of coffee after another. Even now he's fidgeting and unable to concentrate."

\section{Behavioral aspect}

Empathy can also refer to the ability to provide verbal and non-verbal cues that indicate sincere concern and receptiveness. When someone demonstrates empathy, he or she 
communicates interest in the other person and tries to understand in a nonjudgmental and helping manner. Eye contact, a forward lean, and encouraging verbal reinforcers like "I see," or "go on," are examples of how empathy might be communicated.

Empathy is an essential element of relational listening, particularly when members ol a diverse hospitality workforce strive to work together. While earlier models put task accomplishment as the primary goal of effective communication, we now know that relationship aspects are equally as important. The interdependence of workgroups and departments require individuals who understand the person speaking as well as the literal content of the message conveyed.

It might seem as if empathic skills could be learned and then applied at will. This, however, is not necessarily the case. Anyone can prevent another person from understanding him or her; that is, the transactional nature of communication necessitates reciprocity. It may be impossible to understand an employee's perspective, to create a third dimension, if he or she discourages the attempt. Empathy is not something you can force on your partner; it arises out of mutual efforts to understand and be understood, and requires the cooperation of both participants (Broome, 1991).

If, for example, one of your employees appears particularly tired and distracted, you may recognize these cues and make an effort to discover the problem. If, however, this person refuses to discuss the situation with you or intentionally misleads you, understanding will not have been facilitated and your relationship will not have developed.

Barrett-Lennard (1981) proposes that empathy can be approached as a five-step process. The three aspects of empathy presented earlier are all incorporated within this cycle. The first step requires that you assume an appropriate attitude that enables you to be open to the other 
person and that person's unique world view. If, for example, an employee is having difficulty performing what you consider to be a relatively simple task, like learning a new reservation system, it is important to recognize and withhold automatic judgments or assumptions such as, "He could do a better job if he would just try harder," or "He must not have been paying attention during the training sessions."

Barrett-Lennard called the second step empathic resonation. This involves a focused effort to read the other person so that his or her experience becomes clear and vivid. You would imagine the frustration that the employee is feeling over his inability to work with the new equipment, recalling times when you have experienced what you believe are similar feelings.

Step three communicates understanding through verbal and nonverbal behavior, leaning forward, and demonstrating other alternative behaviors. An appropriate verbal response would be something like, "It must be frustrating to have guests become impatient with waiting in line as you try to get the computer program to operate."

Step four is received empathy. The employee now has information regarding the degree to which his manager has understood, and this enables him to complete the cycle by providing feedback to the manager. He may respond by saying, "Yeah, you're right. Sometimes I just want to throw the stupid machine on the floor." In the final stage, understanding is confirmed, corrected, or modified.

Although relational listening is characterized by empathy, there are certain contexts in which empathy is particularly critical. These include when employees are experiencing personal problems, when mistakes are made on the job, or in unusually stressful situations. Keep in mind that empathy is not "feeling what another person feels"; it is, rather, thinking and feeling what you perceive the other person thinks and feels. 


\section{Self-Monitoring}

The final ingredient for effective relational listening is self-monitoring. Although this social skill is, to some extent, personality-linked, all employees can develop their ability to control their behavior in ways that facilitate empathy and the co-creation of meanings. Through self-monitoring, employees increase their sensitivity not only to the verbal and non-verbal cues their partners send, but to the effect their own responses have on the communication situation.

Self-monitoring is "self control guided by situational cues to social appropriateness" (Snyder, 1974, p. 526). Individuals vary in their ability and in their motivation to self-monitor. This competence has significant implications for relational listening when efforts are being made to understand and empathize with those whose backgrounds are dissimilar.

The high self-monitor is sensitive to the nonverbal expressions and indirect cues individuals send during communication, and is particularly conscious of the effect that his or her responses have on others. High self-monitors look to the actions of those around them for cues to determine appropriate behavior. Employees communicate information regarding their status, feelings, and attitudes through nonverbal behavior. The high self monitor attends to these cues and uses them as guides in adjusting his or her own behavior in social situations.

Consider the following situation. Three employees are sitting together in the employee cafeteria. One of them, a Native American, is new to the organization. As she reaches for the salt, she knocks over her iced tea and spills it on her tray and in her soup. The situation has an obvious comic element, and her co-workers respond by laughing. One of them, however, immediately notices that she is not amused; in fact, she is embarrassed and upset. He realizes that laughing is inappropriate in this situation and instantly changes his behavior. 
Low self-monitors, in contrast, rely heavily on their own values, feelings, and attitudes as guides for managing their behavior. A low self-monitor who saw humor in a situation may well continue to laugh; he or she may be unaware of, and so seemingly disregard, the others' feelings and perspective. Consequently, a low self-monitor's communication is relatively consistent and predictable. Although it may sound desirable to work with someone who responds according to his or her true feelings, employees who must get along with individuals unlike themselves are often called upon to modify or to reflect upon their initial response as they work toward the cocreation of meanings. Just as service employees are required to provide emotional labor (Ashforth \& Humphrey, 1993), so too are relational listeners required to respond situationally. Unless individuals take into account the impact their behavior has on others, and respond accordingly, the creation of shared meanings is unlikely.

Snyder's (1974) interest in self-monitoring led him to develop a Sell Monitoring Scale, an 18-item true-false inventory which measures such dimensions as concern for social appropriateness, expressive self-control, and what Snyder termed other-directed sellpresentation. Effective listeners, attend to their partner's behavior in an effort to determine appropriate responses and judge the impact of their own behavior by observing their partner's subsequent reactions. High self-monitors can then assess the effectiveness of the communication strategies they employ and continually modify their performances. In service environments, this behavioral flexibility is not only desirable, it is essential.

\section{Toward Relational Listening: Facilitating Knowledge, Skills, and Attitudes}

For diversity to become an organizational strength, all members must work toward understanding relational listening concepts and developing relational listening attitudes and 
skills. They must value individual differences and recognize the unique contributions of each organizational member. In adopting this perspective, communication must come to be characterized by collaboration, empathy, and high levels of self-monitoring.

Hospitality educators and managers are in an excellent position to foster essential concepts, skills, and attitudes as they interact with future and practicing hospitality employees. Relational listening practices, however, cannot be developed exclusively within a formal training program or classroom; rather, these principles are most meaningful when they become norms of the organization's culture and are regularly addressed within the context of informal, on-going situations.

Educators might foster effective listening not only in the classroom but also during oneon-one interactions with students. If relational listening practices are to become organizational norms, employees must recognize these behaviors as they occur during their daily activities. Within the hospitality workplace, managers might find opportunities through modeling desired behaviors, coaching and counseling employees, and appraising performance.

\section{Training}

Relational listening concepts are appropriately presented within the context of classrooms and training seminars. Although listening can profitably be viewed as an independent topic, the principles are inherently interdisciplinary and can be integrated into a variety of other sessions. The transactional model of communication, the skills of communicating empathy, and the principles of self-monitoring might first be addressed within general orientation sessions or within formal programs on such topics as motivating employees, human relations skills, teambuilding, leadership, managing diversity, or total quality. Participants need to develop a 
common vocabulary to talk about relational listening practices and to understand the impact they have on individual and organizational performance.

Gaining knowledge of these principles, however, is only the first step. In order to create organizational environments that support relational listening practices, employees must also recognize their value and understand their daily application in workplace settings.

\section{Modeling and coaching}

For employees to value and to internalize behaviors that promote healthy relationships and effective communication in diverse environments, managers must themselves demonstrate the principles addressed. This modeling occurs as an on-going activity; managers set standards and establish norms that guide employees' communication practices. Educators can also find opportunities to model these behaviors in and outside of the classroom as they interact with students in a variety of informal, advisement, and instructional settings.

As traditional top-down styles of supervision give way to self-managed teams and employee empowerment, the manager's role becomes one of coach and facilitator, rather than decision-maker and delegator. Coaching is essentially a form of on- the-job training whereby managers work closely and directly with each employee individually to improve job-related skills (Gerber, 1992; Aurelio \& Kennedy, 1991). Coaching is particularly appropriate for the acquisition of relational listening concepts because it is aimed at developing each individual's unique potential.

Through modeling relational listening concepts and providing feedback to the employee during the coaching interview, managers create a collaborative relationship in which the individual employee recognizes the importance of the desired outcomes and actively participates 
in the goal-setting process. Coaching requires a significant shift in the way managers think about their jobs. In the coaching process, the manager and employee collaborate to achieve desired outcomes. The manager practices relational listening behaviors as he or she coaches the employee in developing the necessary skills to communicate effectively within a multicultural organization.

\section{Appraising performance}

If relational listening practices are essential to individual and organizational effectiveness, the degree to which employees demonstrate these attitudes and competencies must be considered in their overall performance appraisal. Norms can only be established and supportive cultures created it reward systems reinforce desired communication practices. The act of talking about these principles is in itself reinforcing: it managers pay attention to their employees' communication behavior and their efforts to collaborate and understand their coworkers, this reinforcement is likely to encourage such behavior to continue.

The appraisal interview also serves as a formal benchmarking opportunity in which the employee's performance is assessed against established personal goals as well as organizational standards. While the coaching process provides on-going feedback and individual support, employees come to understand the importance of relational listening activities when they are an official part of the performance evaluation.

Although the performance appraisal is one of the most significant employee development opportunities, managers can take advantage of other appropriate situations to provide employees with information regarding their application of relational listening concepts. Since such practices have a profound impact on the performance of workgroups and teams, feedback mechanisms 
might be developed to process the group's communication activities, paying special attention to individual reports state to which degree employees felt their ideas were valued and understood by their co-workers.

\section{Conclusion}

Effective communication has always been somewhat problematic in fast-paced hospitality organizations. Today, the challenges have been compounded as the workforce becomes increasingly diverse and as managers realize that equal attention must be paid to the relationship as well as the task dimensions of the job. In recognizing the importance quality of worklife plays in organizational effectiveness, managers seek to maximize the unique contributions of each employee. Organizational members must not only perform tasks, but must also develop supportive and collaborative relationships will) their co-workers.

It is not enough to sensitize employees to the needs of specific minority populations or cultures through diversity training programs. Not only do such sessions risk stereotyping and further alienating various groups, they may not accurately relied the needs and values of each unique member of the particular group.

In an organizational community characterized by diversity, what must remain central and common to all employees is an appreciation of individual differences and an ongoing commitment to the importance of relational listening (Purdy, 1991). In their ongoing activities, each organizational member must communicate effectively with co-workers who have a unique configuration of values, attitudes, beliefs, needs, and competencies. To create cultures that value these individual contributions, managers must encourage every employee to relate to his or her 
colleagues in ways that promote understanding and collaboration (Brownell, 1990; ChristensenHughes. 1992; O’Dwyer. 1992).

Few skills have a more positive impact on communication in the workplace than relational listening. Organizational members who understand communication as a transactional process, who demonstrate empathy in their interactions with others, and who monitor their behaviors create empowering environments that promote the creative and full use of all human resources. As they model these relational listening behaviors, managers themselves may discover that their impact goes beyond improving employee performance to improving the quality of worklife for all organizational members. 


\section{References}

Ashforth, B. E., \& Humphrey, R. H. (1993). Emotional labor in service roles: The influence of identity. Academy of Management Review, 15(1), 88-115.

Aurelio, S., \& Kennedy, J. K., Jr. (1991, August). Performance coaching: A key to effectiveness. Supervisory Management, 1-2.

Barrett-Lennard, G. T. (1981). Dimensions of therapist response as causal factors in therapeutic change. Psychological Monographs, 76(43).

Broome, B. J. (1991). Building shared meaning: Implications of a relational approach to empathy. Communication Education, 40(3), 235-249.

Brownell, J. (1990, Nov/Dec). Managing a diverse workforce: Listening in cross-cultural environments. Asian Hotel and Catering Times, 24-28.

Bruneau, T. (1989). Empathy and listening: A conceptual review and theoretical directions. Journal of the International Listening Association, 3, 1-20.

Christensen, J. (1993). The diversity dynamic: Implications for organizations in 2005. Hospitality Research Journal: The Futures Issue, 17(1), 69-86.

Christensen-Hughes, J. (1992). Cultural diversity: The lesson of Toronto's hotels. Cornell Hotel \& Restaurant Administration Quarterly, 33(2), 78-87.

Del Sesto, C. (1993, May). Women in lodging: How far have they come? Lodging, 28-32.

DeMicco, F. J., \& Reid, R. D. (1988). Older workers: A hiring resource for the hospitality industry. The Cornell Hotel \& Restaurant Administration Quarterly, 28, 56-61.

Gamio, M. O., \& Sneed, J. (1992). Cross-cultural training practices and needs in the hotel industry. Hospitality Research Journal, 15(3), 13-26. 
Gerber, D. A. (1990). Listening to the disabled people. Disability, Handicap and Society, 5(1), 323.

Gerber, B. (1992, February). From manager into coach. Training, 25-31.

Greenslade, M. (1991, December). Managing diversity: Lessons from the US. Personnel Management, 23(12), 28-33.

Griffin, E. (1992). Meetings and cultural diversity. Meetings \& Conventions, 27(2), 22.

Mill, R. C. (1994). Cross-cultural communication: An integrated approach. Hospitality \& Tourism Educator, 5(1), 67-70.

O’Dwyer, C. (1992). Special Report: Opening doors and minds. Lodging Magazine, 17(9), 1215.

Powers, T. F. (1992). Managing international hospitality. Hospitality Review, 19(1), 41-51.

Purdy, M. (1991). Listening and community: The role of listening in community formation. Journal of the International Listening Association, 5, 51-67.

Sillies, P. J., DeMicco, F. J., Kavanaugh, R. R., \& Mann, S. H. (1994). Attitudes of hospitality management students toward older workers: A follow-up study. Hospitality \& Tourism Educator, 6(1), 7-15.

Smith, J. H,, Jr. (1992). A study of handicapped employment in the hospitality industry. The Hospitality \& Tourism Educator, 4(3), 16-25.

Snyder, M. (1974). Self-monitoring of expressive behavior. Journal of Personality and Social Psychology, 30(4), 526-537.

Woods, R. H,, \& Kavanaugh, R. R. (1992). Here comes the ADA-Are you ready? (Part I). Cornell Hotel \& Restaurant Administration Quarterly, 33(1), 24-32. 


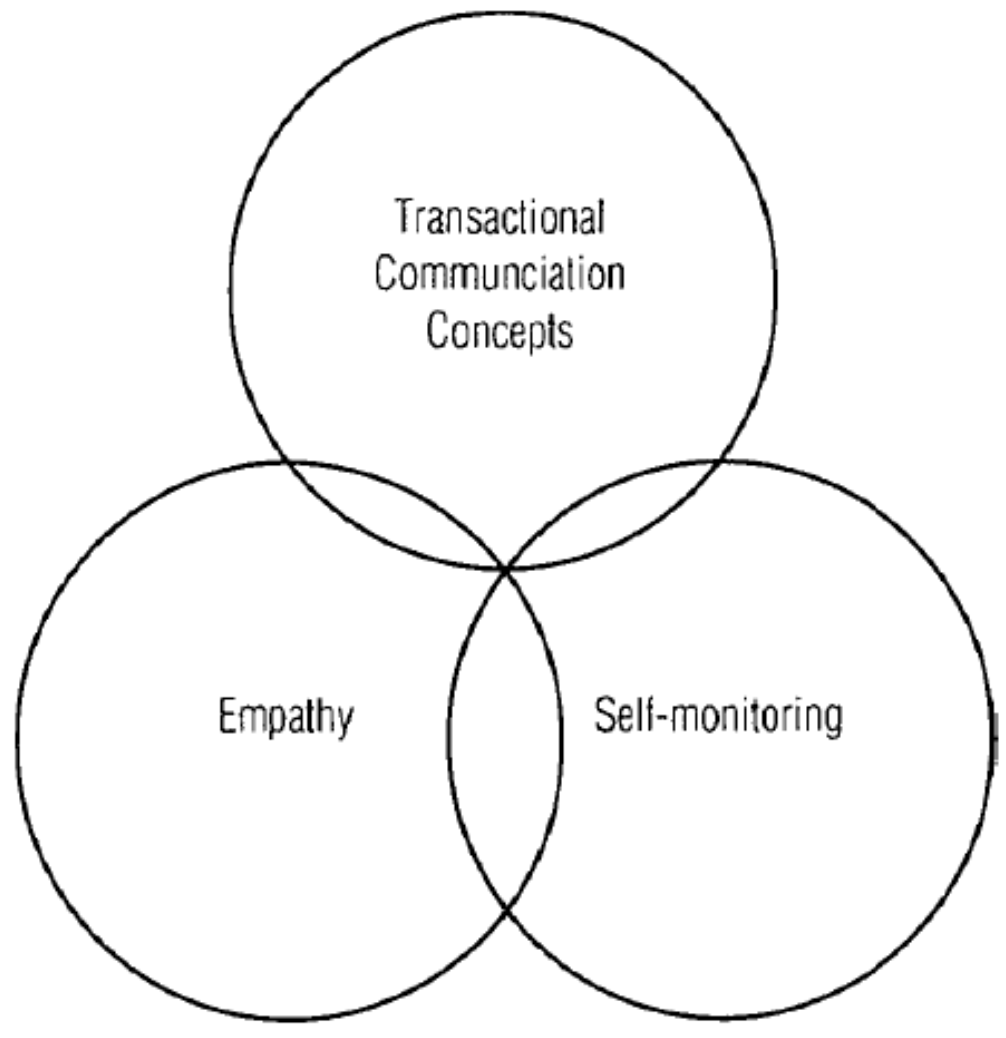

Figure 1. Relational Listening 

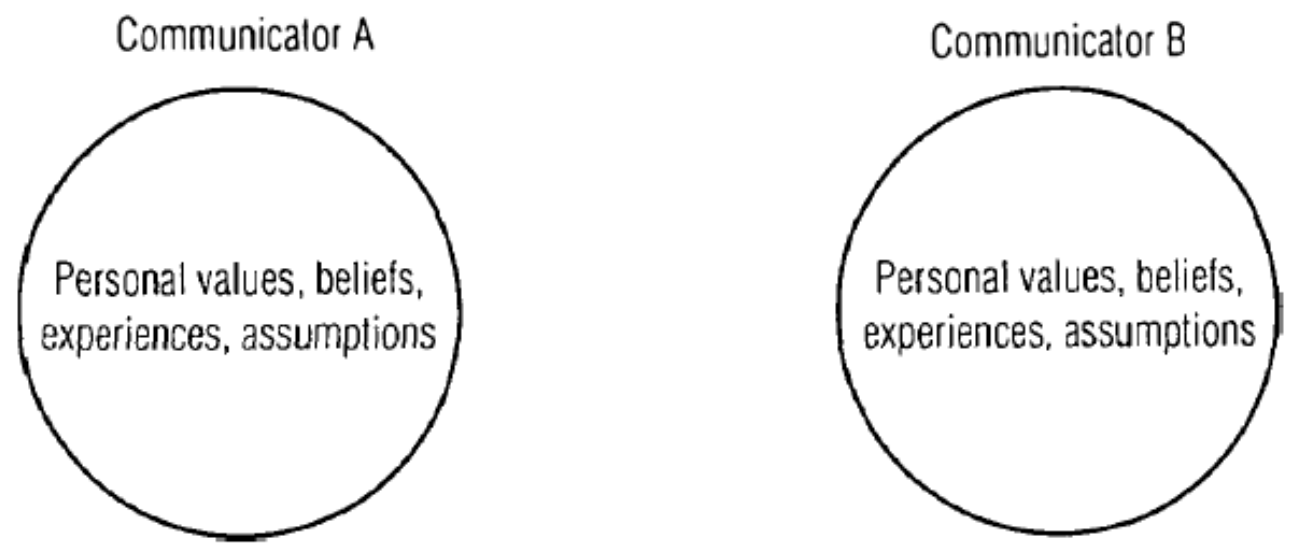

Withoul common backgrounds, communicators have no shared experiences and no common understandings.

Communicator A Communicator B

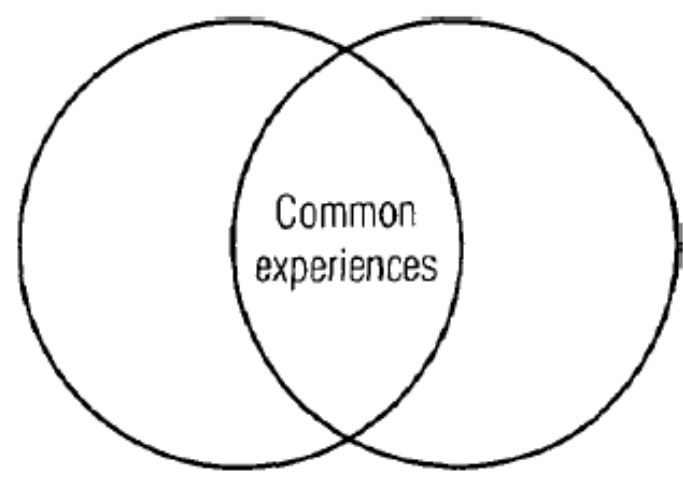

Communication is effective to the extent that communicalor's backgrounds overlap.

Figure 2. Traditional View of Communication. 


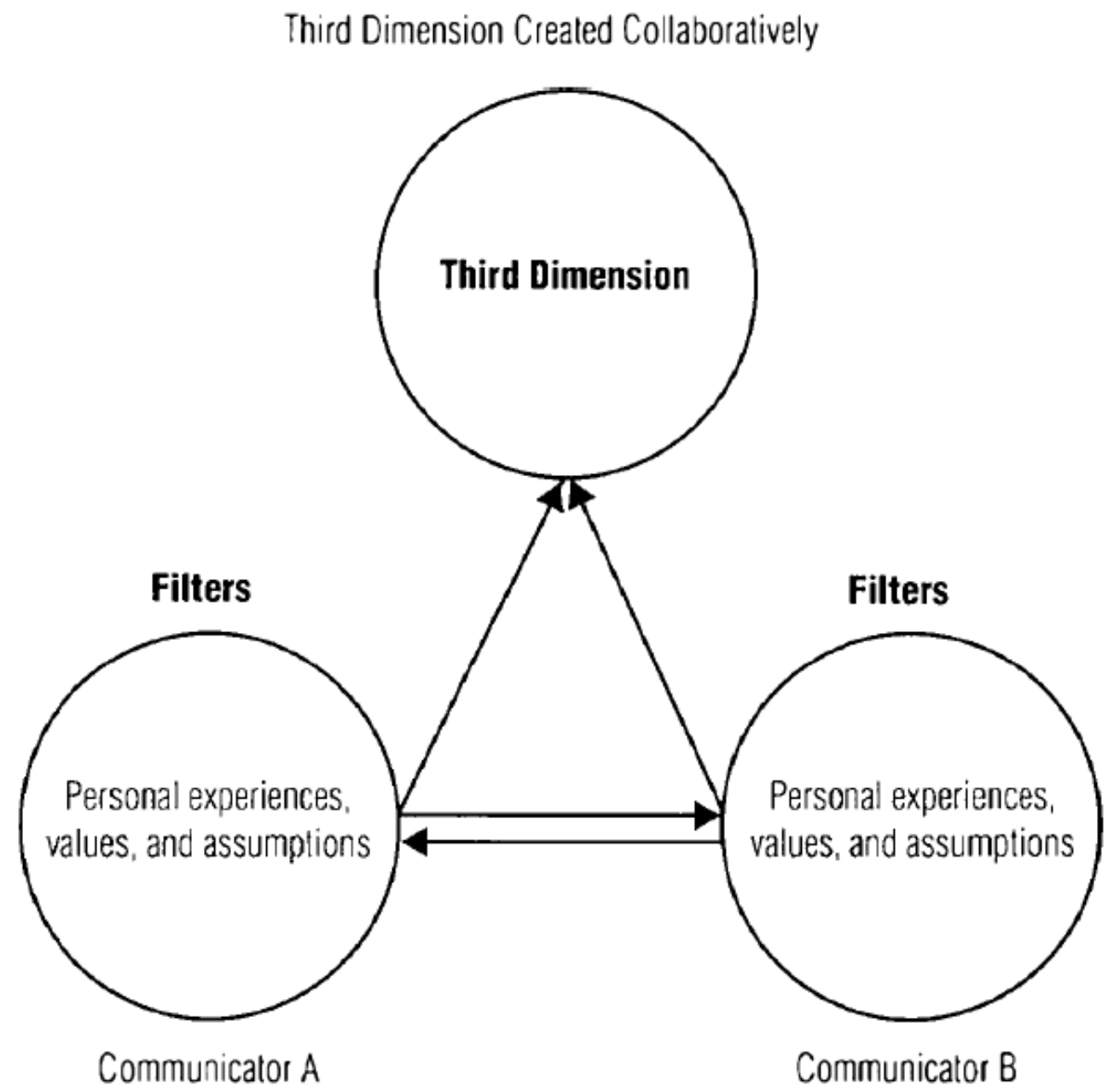

Figure 3. Transactional Model of Communication 